\title{
INTERFUEL: FAST - Updates for FY 2021 Reporting
}

\section{September 2021}

Ron Stewart
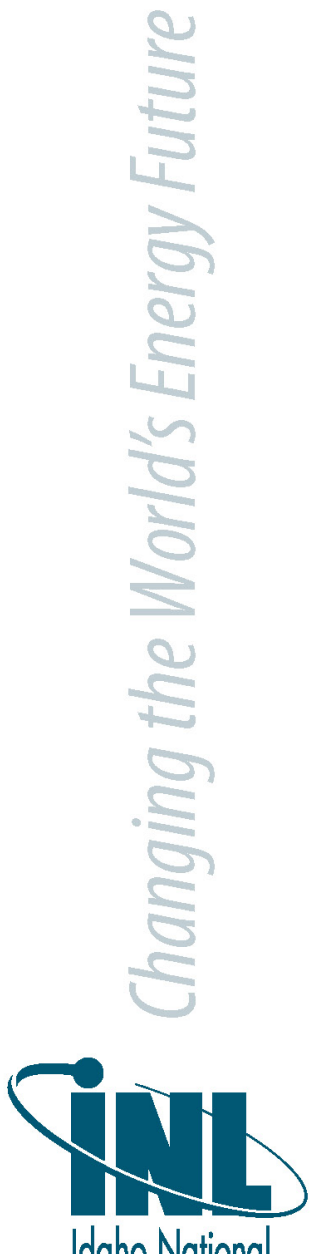

Idaho National

Laboratory 


\section{DISCLAIMER}

This information was prepared as an account of work sponsored by an agency of the U.S. Government. Neither the U.S. Government nor any agency thereof, nor any of their employees, makes any warranty, expressed or implied, or assumes any legal liability or responsibility for the accuracy, completeness, or usefulness, of any information, apparatus, product, or process disclosed, or represents that its use would not infringe privately owned rights. References herein to any specific commercial product, process, or service by trade name, trade mark, manufacturer, or otherwise, does not necessarily constitute or imply its endorsement, recommendation, or favoring by the U.S. Government or any agency thereof. The views and opinions of authors expressed herein do not necessarily state or reflect those of the U.S. Government or any agency thereof. 


\title{
INTERFUEL: FAST - Updates for FY 2021 Reporting
}

\author{
Ron Stewart
}

September 2021

\section{Idaho National Laboratory \\ Idaho Falls, Idaho 83415}

http://www.inl.gov

\author{
Prepared for the \\ U.S. Department of Energy \\ Under DOE Idaho Operations Office \\ Contract DE-AC07-05ID14517, DE-AC07-05ID14517
}




\section{FAST: Updates for FY 2021 Reporting}

September 2021 INTERFUEL Meeting 


\section{Updates to Fleet Data Reporting}

- Expanded set of vehicle types now valid as EV / PHEV fuel configurations

- ... and additional types available for future-year acquisition plans

- Updated limits for many operational cost-related validation rules

- Improved year-to-year vehicle matching for consistency validation rules **

- Updated validation rules for future-year fleet costs

- Revised blocking, new flagging limit on age of planned owned disposals

- Additional rules for planned cost consistency across fleet segments * 


\section{Updates to FAST's Query Tool}

- New query types for fueling center and EVSE inventory data

- Updated start page for query tool:

- Categorized query types to emphasize current data submissions

- Additional detail in query type descriptions

- Additional filtering capabilities **

- Additional vehicle attributes available

FAST Query Tool - Query Types

Vehicle-Level Data (VLD) Information

vehicles

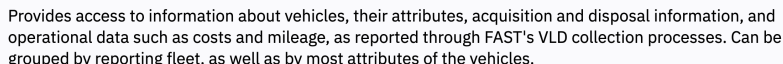

Note: This information is avaiable only for Fr 2016 and Iater years, depending on when individual agencies
transitioned to reporting fleet data as VLD. See "Section 1 : Inventory, Acquistition, and Disposal Data" in the Fuel.

validation Flass

Future-Year Acquisition and Disposal Plans

Future-Year Fleet Cost Plans

fueling Center and EVSE Inventory Information

Fueling center Data

Electric Venicle Supply Equipment(EVSE)Inventory Data

FAST System Information

User Information

istorical / Legacy FAST Data

Section 1: Inventory,Accquisition, and Disposal Data

Section 2: Fleet Costs and Mileage Data :

Section 3: Fuel Costs and Consumption Data -

EPAct $\$ 701$ Waiver Request Data

EPAct $\$ 701$ Waiver Request Review Data

Legacy Electric Vehicle Supply Equipment Inventory

Legacy EISA S 246 Fueling Inffrastructure Data : 


\section{Fueling Center \& EVSE Inventory Reporting}

- Reporting based on previous year's submission

Fueling Center and EVSE Inventory Reporting

- Agency POC downloads pre-filled Excel submission template

- Agency updates fueling centers, EVSE inventory

- Update previously-submitted entries (status, POC, fuel types / \# of EVSE)

- Designate closed entries

- Add new entries, if any

- Agency POC imports to FAST for validation

- Agency POC designates submission as complete

Department of Energy - FY 2021

Reporting Resources $\oplus$

- FAST Help: Fueling Center and EVSE Inventory Reporting

- Generate and download pre-filled FY 2021 reporting template for Department of Energy...

Upload Fueling Center and EVSE Inventory

Excel File: Browse... No file selected.

Note: Validating and processing data may take several minutes, depending on the amount of data being processed and current activity on the system's servers. Please be patient!

Upload Reset Form

Submission Status

Summary: 0 fueling center entries and 0 EVSE entries submitted Status: $\odot$ Incomplete $\bigcirc$ Complete

Update Submission Status Reset Form 


\section{Additional Updates}

\section{- VLD Export}

- Agencies can now generate Excel exports of past VLD submissions (vehicles + fuel or future year projections)

- XML export to be implemented in future **

- Valid Vehicle Type + Fuel Configuration Report **

- Per agency, per year report showing valid combinations

- Also shows valid EISA 2005 Section 141 designations for each vehicle type + fuel configuration

- Improved Query Tool Export Capability **

- Excel exports will include information about filtering, hidden columns, etc., used to build the query 


\section{Reporting Timeline}

\section{- September: Preparation}

- Agencies start fueling center \& EVSE inventory reporting

- FAST team will host on-line sessions for agencies on process

- Submissions due mid-November

- FY 2021 data call preparation

- Hierarchy, budget element codes, user account updates

- Updated reporting references published (late September)

\section{- October 1 - December 15: Data Call}

- Agencies complete FY 2021 submission

\section{- January-February: Review and Feedback}

- FAST team + agency review, feedback, follow-up cycle 


\section{Recommendations}

\section{- Start early}

- ... and finish early for preliminary review feedback from FAST team

- Review feedback received after last year's submission

- Take advantage capabilities in FAST

- Import in "validate" mode

- Use FAST Sandbox

- Use flagging validation rules, reports, and query tool to investigate potential quality issues 


\section{We Can Help}

\section{FASTsupport@inl.gov}




\section{FAST Program Points of Contact}

- DOE Federal Energy Management Program

- Lay Wrobel

Jay.Wrobel@ee.doe.gov

- GSA Office of Government-wide Policy

- Wnes Vogelsinger

James.Vogelsinger@gsa.gov
- FAST Support Team

- FASTsupport@inl.gov

- @ @FASTdevs

- FAST

- https://fastweb.inl.gov/

- FAS 马ुHelp

- https://fastweb.inl.gov/help/ 


\section{Ron Stewart}

FAST Team Lead

Idaho National Laboratory

ron.stewart@inl.gov 